\title{
ON THE TRAGE OF THE ENERGY-MOMENTUM TENSOR OF FIELDS ASSOGIATED WITH PARTICLES OF ZERO REST MASS
}

\author{
H. A. BUCHDAHL * \\ (rec. 8 August 1958)
}

\begin{abstract}
Summary
The trace $T$ of the metrical energy-momentum tensor $T_{k}{ }^{l}$ of fields associated with particles of zero rest mass may be zero, either identically or as a consequence of the field equations. This property of $T^{k l}$ is correlated here with the behaviour of the Lagrangian of the field under arbitrary conformal transformations. Certain classes of special fields are considered explicitly. It is shown in particular that $T$ vanishes for all non-zero spin fields which correspond respectively to the two-component neutrino field or the photon field.
\end{abstract}

\section{Introduction}

In a recent paper Brill and Wheeler [1] derive the explicit form of the metrical energy momentum tensor $T^{k l}$ of the neutrino field and add the comment that it is remarkable that its trace vanishes [as a consequence of the field equations] just as it does for the photon field. In the case of the latter the trace $T\left(=T_{l}^{l}\right)$ of course vanishes identically, i.e. even when the field equations are not satisfied. In this paper I now show that the vanishing or otherwise of $T$ in the case of general fields (of which the two fields just mentioned are special examples) depends simply upon the conformal behaviour of the Lagrangian density $\mathfrak{L}$ of the field. A general theorem to this effect is derived in section II. Arbitrary infinitesimal variations of the metrical tensor and of the linear connections in world and in spin space, along with their behaviour under arbitrary conformal transformations are considered in the next two sections. Finally, in sections $\mathrm{V}$ and VI the property of the neutrino field referred to above is shown to be possessed by wide classes of tensor and spinor fields; and the cases of non-zero rest mass and of non-linear field equations are briefly referred to.

* Present address: Institute for Advanced Study, Princeton, New Jersey. 


\section{A General Theorem}

(a) In so far as spinor fields enter into the present considerations the notation employed will be that of the two-spinor analysis of Infeld and van der Waerden [2]. A "general" field shall be described by certain field quantities $\zeta_{(1)}, \zeta_{(2)}, \ldots$ (all indices suppressed) which may be tensors, spinors, or tensor-spinors of any kind. (Thus for example, $\zeta_{(1)}$ and $\zeta_{(2)}$ might stand for the spin vectors $\xi_{\mu}, \eta^{\dot{\mu}}$ of the Dirac field and $\zeta_{(3)}$ for the vector of the photon field, i.e. the electromagnetic potential; etc.) The index $s$ which distinguishes the $s$ 'th component fields $\zeta_{(s)}$ shall be excluded from the summation convention. It is convenient to take the $\zeta_{(s)}$ as associated with the points of a Riemann space $V_{4}$, whose metrical tensor is $g_{k l}$. The $V_{4}$ will be regarded as fixed, that is, in so far as one may regard the $g_{k l}$ as the components of the gravitational potential any reaction of the fields under consideration upon the gravitational field shall be disregarded. Alternatively one may regard the introduction of the $V_{\mathbf{4}}$ as merely a temporary expedient; after the infinitesimal variations and conformal transformations contemplated below have been carried into effect one returns to Galilean space-time by taking the untransformed metrical tensor to be Galilean. One is in any case virtually forced to this viewpoint as soon as one attempts to treat fields for spin $\geqq \frac{3}{2}$ in an arbitrary $V_{4}$ (cf. Buchdahl [3].)

The field equations will be supposed to arise from a real Lagrangian \&, that is, - apart possibly from the presence of certain subsidiary conditions, - they shall have the form

$$
Z_{(s)}=0, \quad(s=1,2, \ldots)
$$

where $Z_{(s)}$ is the hamiltonian derivative of $\mathfrak{R}$ with respect to $\zeta_{(s)}$. Again, the metrical energy-momentum tensor $T^{k l}$ is the hamiltonian derivative of $\mathfrak{L}$ with respect to $g_{k l}$ (see section 3 ); and by considering variations of $g_{k l}$ which arise from infinitesimal variations of coordinates one obtains in the usual way the identity

$$
T_{; l}^{k l}=0,
$$

which allows one to formulate the usual conservation laws in galilean spacetime in a straightforward manner.

Now, the $Z_{(s)}$ and $T^{k l}$ being hamiltonian derivatives one has identically for all simultaneous variations of the $\zeta_{(s)}$ and $g_{k l}$ which vanish on the boundary $\Omega$ of the region of integration

$$
\delta \int \mathcal{L} d \tau=\int\left(T^{k l} \delta g_{k l}+\sum_{s} Z_{(s)} \delta \zeta_{(s)}\right)(-g)^{\frac{1}{2}} d \tau
$$


(b) A general conformal transformation of $V_{4}$ is the passage from $g_{k t}$ to ' $g_{k l}$,

where

$$
{ }^{\prime} g_{k l}=\lambda^{2} g_{k l}
$$

$\lambda$ being an arbitrary real function of the coordinates. With every $\zeta_{(s)}$ there shall now be associated a "conformal weight" $c_{s}\left(c_{s}\right.$ real), and a conformal transformation of the field shall mean the transformation (2.4) coupled with the simultaneous replacement of $\zeta_{(s)}$ by ' $\zeta_{(s)}$, where

$$
\zeta_{(s)}=\lambda^{c s} \zeta_{(s)} \text {, }
$$

(see sections 4 and 5 ). In particular any quantity is to be called a conformal invariant if conformal transformations of the field leave it unaffected. Thus $\zeta_{(s)}$ is a conformal invariant if its conformal weight is zero.

Now it may so happen that the $c_{s}$ can be chosen in such a way that the Lagrangian density $\Omega$ becomes a conformal invariant. When this is the case it follows that in an arbitrary infinitesimal conformal transformation which is zero on $\Omega,(\lambda=1+\eta$, say, where $\eta$ is infinitesimal)

$$
\delta \int \mathfrak{L} d \tau=\int\left(T^{k l} g_{k l}+\sum_{s} c_{s} Z_{(s)} \zeta_{(s)}\right) \eta(-g)^{\frac{1}{2}} d \tau
$$

Since $\eta$ is arbitrary within $\Omega$ this implies

$$
T=-\sum_{s} c_{s} Z_{(s)} \zeta_{(s)}
$$

One therefore has the general result that if the Lagrangian density of the field is a conformal invariant then the trace $T$ of the energy-momentum tensor vanishes: it does so as a consequence of the field equations unless the field quantities themselves are conformal invariants, in which case $T$ vanishes identically.

\section{Variations of $g_{k l}$}

This section deals briefly with the effects of general variations of $g_{k l}$. A variation $h_{k l}\left(=\delta g_{k l}\right)$ of $g_{k l}$ induces a variation of the linear connection of $V_{4}$, i.e. of the Christoffel symbols $\Gamma_{a b}^{c}$, given by

$$
\delta \Gamma_{a b}^{c}=\frac{1}{2}\left(h_{a ; b}^{c}+h_{b ; a}^{c}-h_{a b ;}^{c}\right),
$$

as is easily confirmed. The basic vector spinor $\sigma^{k \mu \nu}$ varies in such a way that

$$
\delta\left(\sigma^{k \dot{\mu} \boldsymbol{\nu}} \sigma_{\dot{\mu} \nu}^{l}\right)=-h^{k l} .
$$

It suffices to take (see the remarks following eq. (3.4))

$$
\delta \gamma_{\mu \nu}=0
$$


in which case $(3.2)$ is satisfied by

$$
\delta \sigma^{k \dot{\mu} \nu}=-\frac{1}{2} h_{a}^{k} \sigma^{a \dot{\mu} \nu} .
$$

Upon the solution $(3.3,4)$ of $(3.2)$ one may superpose an arbitrary infinitesimal spin transformation $\Lambda^{\alpha}{ }_{\beta}$. In this way one obtains the most general variations of the $\sigma^{k \mu \nu}, \gamma_{\alpha \beta}$ compatible with $h_{a b}$ since they are specified by $16+2$ real functions, a number equal to the $10+8$ independent real functions involved in $h_{a b}, \Lambda^{\alpha}{ }_{\beta}$. Though it may at times be convenient to draw upon such additional spin transformations $(3.3,4)$ are sufficiently general in the present context.

Taking $\delta \Gamma_{\lambda k}^{\lambda}=0$ one may now derive from the equation

$$
\delta\left(\sigma_{; k}^{l \mu \nu}\right)=0
$$

the variation of the linear spinor connection, viz.

$$
\delta \Gamma^{\nu}{ }_{\mu k}=\frac{1}{2} S^{a b \nu}{ }_{\mu} h_{k a ; b},
$$

where

$$
S_{\mu}^{a b \nu}=\sigma_{\dot{\lambda} \mu}^{[b} \sigma^{a] \dot{\lambda} \nu} .
$$

Given $\mathfrak{L}$ in any particular case the explicit expression for $T^{a b}$ may then easily be derived using eqs. (3.1), (3.4) and (3.6).

\section{Conformal Transformations}

The transformations of the basic spinors associated with the conformal transformation

$$
{ }^{\prime} g_{k l}=\lambda^{2} g_{k l}
$$

of the $V_{4}$ will be taken as

$$
\begin{gathered}
{ }^{\prime} \sigma^{k \mu \nu}=\lambda^{-1} \sigma^{k \dot{\mu} \nu}, \\
{ }^{\prime} \gamma_{\mu \nu}=\gamma_{\mu \nu} .
\end{gathered}
$$

(4.3) corresponds to the choice (3.3). The most general associated transformation can be obtained from $(4.2,3)$ by superposing upon them a spin transformation of the form

$$
\Lambda_{\mu}{ }^{\nu}=\delta_{\mu}{ }^{\nu} \lambda^{c}
$$

and the choice $c=+\frac{1}{2}$ is indeed often convenient in other contexts. If subscripts following a colon indicate covariant differentiation with respect to the transformed linear connections, then

$$
\sigma^{k \dot{\mu \nu}}: s=0 \quad\left({ }^{\prime} \gamma_{\dot{\alpha} \dot{\beta}}{ }^{\prime} \gamma_{\mu \nu}\right)_{: s}=0
$$


It is convenient to write $\lambda=e^{q}$, and, for either linear connection, ' $\Gamma=\Gamma+\Delta$ (indices suppressed.) Then

$$
-\sigma^{k \dot{\mu} \nu} q_{, s}+\Delta_{s t}^{k} \sigma^{t \dot{\mu} \nu}+\Delta_{\dot{\alpha} s}^{\dot{\mu}} \sigma^{k \dot{\alpha} \nu}+\Delta_{\alpha s}^{\nu} \sigma^{k \dot{\mu} \alpha}=0,
$$

and

$$
\Delta_{\epsilon s}^{\epsilon}+\Delta_{\dot{\varepsilon} s}^{\dot{\varepsilon}}=0
$$

It is natural to take $\Delta^{\epsilon}{ }_{\epsilon s}=0$. Using

$$
\Delta^{s}{ }_{k l}=\delta^{s}{ }_{k} q_{; l}+\delta^{s}{ }_{l} q_{; k}-g_{k l} q^{; s}
$$

(4.6) then yields easily

$$
\Delta^{\nu}{ }_{\mu s}=S_{s}^{b \nu}{ }_{\mu} q_{; b} .
$$

(4.8) will be seen to be consistent with (3.7) on taking $q$ to be infinitesimal and setting $h_{k l}=2 q g_{k l}$.

\section{Special Fields}

(i) The two-component neutrino field. This field may be taken as a prototype for the case of half odd-integral spin. The Lagrangian is

$$
\mathfrak{L}=\frac{1}{2} i \sigma^{k \dot{\mu \nu}}\left(\zeta_{\dot{\mu}} \zeta_{\nu ; k}-\zeta_{\nu} \zeta_{\dot{\mu} ; k}\right)=\frac{1}{2} i(l-\bar{l}) \text {, say. }
$$

Then in a conformal transformation

$$
' l=e^{(c-1) q} \sigma^{k \dot{\mu} \nu} \zeta_{\mu^{*}}\left(e^{c a} \zeta_{\nu}\right)_{: k},
$$

where $c$ is the conformal weight of $\zeta_{\nu}$. Using (4.8) this becomes

$$
' l=e^{(2 c-1) q} \sigma^{k \dot{\mu} \nu} \zeta_{\mu}\left(\zeta_{\nu ; k}+c q_{, k}-S_{k}{ }^{b \lambda}{ }_{\nu} q_{, b} \zeta_{\lambda}\right)
$$

Now

$$
\sigma^{k \dot{\mu} \nu} S_{k}^{b \lambda}{ }_{\nu}=-\frac{3}{2} \sigma^{b \dot{\mu} \lambda}
$$

so that

$$
\mathfrak{l}=(-g)^{\frac{1}{2}} e^{(2 c+3) q}\left[l+\left(c+\frac{3}{2}\right) \sigma^{b \dot{\mu} \nu} \zeta_{\dot{\mu}} \zeta_{\nu} q_{, b}\right] .
$$

Accordingly, taking $c=-\frac{3}{2}$, the Lagrangian density will be a conformal invariant, and since $c \neq 0$ it follows that in this case $T$ vanishes, but only as a consequence of the field equations. Indeed, using (3.7) and keeping in mind that the field equations are

$$
\sigma^{k \dot{\mu} \nu} \zeta_{\nu ; k}=0 \text {, }
$$

it is not difficult to show that $T_{k l}$ is equal to the symmetrical part of the imaginary part of $-\frac{1}{2} \sigma_{k}{ }^{\dot{\mu} \nu} \zeta_{\dot{\mu} ; \imath} \zeta_{\nu}$; and the trace of the latter is zero in virtue of (5.5).

(ii) The spin $s-\frac{1}{2}(s=1,2, \ldots)$ field analogous to the two-component neutrino field. 
The Lagrangian may be taken as $L=\frac{1}{2} i(l-l)$, where

$$
l=\sigma_{\nu_{1}}^{k \dot{\lambda}} \zeta^{\dot{\mu}_{1} \ldots \mu_{s-1} \nu_{1} \ldots \nu_{s}} \zeta_{; k} \ldots \nu_{s} \dot{\lambda}_{\mu_{1} \ldots \dot{\mu}_{s-1}}
$$

which may, for later convenience, be written in the symbolic form $\sigma_{\nu_{1}}^{k \dot{\lambda}} \zeta_{; k} \zeta^{*}$. The field spinor $\zeta$ is symmetric in all its dotted and all its undotted indices, I shall merely indicate in outline the conformal transformation of $l$. In the expression analogous to (5.2) there will again be a factor $e^{(2 c-1) a}$, if $c$ is the conformal weight of $\zeta$. On the other hand there will now be $2 s-1$ terms corresponding to the last term on the right hand side of (5.2). Upon simplification in the manner above one finds that (a) the $s-1$ dotted indices of $\zeta_{; k}$ contribute $-\frac{1}{2}(s-1) Q$, where $Q=\sigma^{\dot{k} \lambda}{ }_{\nu_{1}} q_{; k} \zeta \zeta^{*}$, (b) the first undotted index contributes $+\frac{3}{2} Q$, (c) the remaining $s-1$ undotted indices contribute $+\frac{1}{2}(s-1) Q$. Hence the term corresponding to the last one of $(5.4)$ has again a factor $c+\frac{3}{2}$. Therefore, with $c=-\frac{3}{2}, T$ vanishes for all such fields, but only as a consequence of the field equations.

(iii) Integral spin fields analogous to the photon field.

In the case of a field corresponding to particles of spin $s(s=1,2, \ldots)$ one may take the field variable to be a completely symmetrical tensor of zero trace and zero divergence:

$$
A_{k a_{3} \ldots a_{s}}^{k}=0, \quad A_{a_{2} \ldots a_{s} ; k}^{k}=0 .
$$

The Lagrangian takes the form

$$
\mathfrak{L}=A_{\left[k\left|a_{2} \ldots a_{2}\right| ; l\right]} A^{\left[k\left|a_{2} \ldots a_{2}\right| ; l\right]} .
$$

Write the first and second factors on the right in the abbreviated forms $f_{k l}, f^{k l}$ respectively. Then it is easily confirmed by means of $(4.7)$ that the conformal transform of $f_{k l}$ is

$$
\begin{aligned}
f_{k l}=e^{c q}\left\{f_{k l}+\right. & (c-s+1) q_{;[l} A_{k] a_{2} \ldots a_{s}} \\
& \left.+\sum_{t=2}^{s} g_{a_{t}[l} A_{k] \ldots a_{t-1} b a_{t+1} \ldots a_{s}} q^{; b}\right\},
\end{aligned}
$$

$c$ being the conformal weight of $A$. When ' $f_{k l}$ is multiplied by ' $f^{k l}$ the sum over $t$ in (5.9) may evidently be rejected, because of (5.7). Since $f^{k l}$ arises

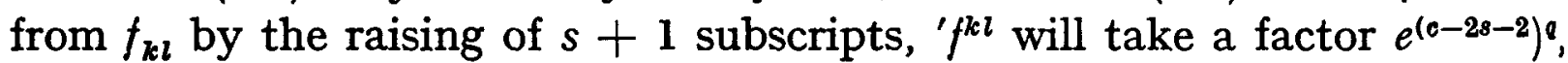
whilst $\mathbb{Q}$ will take a factor $e^{(2 c-2 s-2+4) q}=e^{2(c-8+1) q}$. But the numerical coefficient of $2 q$ in the exponent is just that which also occurs in the second term on the right hand side of (5.9). Hence, taking $c=s-1$, the Lagrangian density is a conformal invariant. It follows that $T$ vanishes for all $s>1$ as a consequence of the field equations, whilst for $s=1$ it vanishes identically. (The latter is of course a well known property of the photon field.) It may be noted that the case $s=0$ stands apart from all these in that the Lagrangian density is essentially not an invariant under the general conformal group. 


\section{Conclusion}

The general result of $\S 2$ has made it possible to infer in a straightforward manner that the trace of the energy-momentum tensor vanishes for a wide class of fields corresponding to particles of zero rest mass and arbitrary spin, to which the case of zero spin is the only exception. Moreover, with the sole exception of spin $1 T$ vanishes only as a consequence of the field equations.

At the same time it is immediately clear why the situation is different for fields associated with particles of non-zero rest mass. Taking the case of the tensor fields of $\S 5$ (iii) for example the Lagrangian density will contain an additional term

$$
\kappa^{2} A_{k a_{2} \ldots a_{8}} A^{k a_{2} \ldots a_{s}}(-g)^{\frac{1}{2}}
$$

where $\kappa$ is a constant. In a conformal transformation this takes the factor $e^{2(c-s+2) q}=e^{2 q}$; and the Lagrangian density as a whole consequently cannot be a conformal invariant.

The methods and conclusions above are not essentially altered if more complicated, or several interacting, fields be contemplated. However, if one drops the requirement that the field equations be linear, then the possibility of constructing conformally invariant Lagrangians is greatly extended. Taking again the example of the tensor fields of $\S 5$ (iii) one will obtain a field for which $T=0$ and whose equations are quasi-linear if one adds to the Lagrangian above for instance the term

$$
\left(\kappa A_{k a_{2} \ldots a_{s}} A^{k a_{2} \ldots a_{s}}\right)^{2}
$$

where $\kappa$ is a constant.

\section{References}

[1] Brill, D. R. and Wheeler, J. A., Interaction of neutrinos and gravitational fields, Revs. Mod. Phys., 29 (1957), 465-479.

[2] Infeld, L. and Van der Waerden, B. L., Die Wellengleichung des Elektrons in der allgemeinen Relativitätstheorie, Sitz. Ber. der preu. Akad. Wiss., (1933), 380-401.

[3] Buchdahl, H. A., On the compatibility of relativistic wave equations for particles of higher spin in the presence of a gravitational field, Nuovo Cimento, - 10 (1958), 96-103.

Physics Department, University of Tasmania, Hobart, Tasmania. 